\title{
Polyetheretherketone (PEEK) Dental Implants: A Case for Immediate Loading
}

\author{
${ }^{1}$ Karan Marya, ${ }^{2} \mathrm{JS}$ Dua, ${ }^{3}$ Sonia Chawla, ${ }^{4}$ Priyanshu R Sonoo, ${ }^{5}$ Anuj Aggarwal, ${ }^{5}$ Veenita Singh \\ ${ }^{1}$ Professor, Department of Oral and Maxillofacial Surgery, Santosh Dental College and Hospital, Santosh University \\ Ghaziabad, Uttar Pradesh, India \\ ${ }^{2}$ Associate Professor, Department of Oral and Maxillofacial Surgery, Santosh Dental College and Hospital, Santosh University \\ Ghaziabad, Uttar Pradesh, India \\ ${ }^{3}$ Reader, Department of Oral and Maxillofacial Surgery, Santosh Dental College and Hospital, Santosh University \\ Ghaziabad, Uttar Pradesh, India \\ ${ }^{4}$ Senior Lecturer, Department of Oral and Maxillofacial Surgery, Santosh Dental College and Hospital \\ Santosh University, Ghaziabad, Uttar Pradesh, India \\ ${ }^{5}$ Postgraduate Student, Department of Oral and Maxillofacial Surgery, Santosh Dental College and Hospital \\ Santosh University, Ghaziabad, Uttar Pradesh, India
}

Correspondence: Kiran Marya, Professor, Department of Oral and Maxillofacial Surgery, E-319, GF, Greater Kailash 2, New Delhi, India, e-mail: marya_dental@yahoo.com

\section{ABSTRACT}

In the last three decades, polyetheretherketone (PEEK) has been increasingly employed as a biomaterial for orthopedic and spinal implants. PEEK dental implants have shown equal promise and are currently being used and investigated in many parts of the world. While their osseointegration capacity has been established beyond doubt, it is not clear whether these implants are suitable for specific situations with low functional and high esthetic demands or as completely viable alternatives to titanium in all situations. We present a brief introduction to the PEEK implants, a representative system and kit and the advantages and disadvantages as compared to titanium implants.

It is our observation that PEEK implants function well in anterior as well as posterior regions and when loaded immediately (one week after placement). Their use in the esthetic zone can be of significant advantage to the surgeon as well as patient. However, diminished radiopacity and limitation of available sizes may be causes for concern.

Keywords: Polyetheretherketone (PEEK), Biopik, Endosseous implants.

\section{INTRODUCTION}

For decades the universal choices for implant material have been titanium and its alloys. Often tagged as the wonder metal, titanium's strength, low weight, corrosion resistance and biological inertness made it the prime candidate for making 'fit and forget' medical implants. However, the search for a material that is more ideally suited for this purpose has intensified in the last few years. This is partially owing to some potential disadvantages of titanium as well as addressing some practical clinical issues.

Presently most commercially available implant systems are made of pure titanium (CP-Ti) or titanium alloy (Ti-6Al-4V, i.e. titanium-aluminum-vanadium). While pure titanium has mechanical properties similar to type III and type IV gold alloys, the titanium alloy castings have properties closer to $\mathrm{Ni}-\mathrm{Cr}$ and $\mathrm{Cr}$-Co castings with the exception of lower modulus. ${ }^{1}$ Even though both CP-Ti and Ti alloys are exceptionally corrosion resistant because of the stability of the $\mathrm{TiO}_{2}$ layer, they are not inert to corrosive attack. When the stable oxide layer is broken down or removed and is unable to reform on part of the surface, titanium can be as corrosive as many other base metals. ${ }^{2}$ Galvanic coupling of titanium to other restorative metals may also generate corrosion. Hence, there is a great concern regarding the materials for suprastructures over the implants. While corrosion leads to weakening of the implant structure, liberation of corrosion elements can produce osteolysis ${ }^{3}$ and marginal bone loss. ${ }^{4}$ All of these may result in implant failure with the clinician left clueless as to the cause of its occurrence.

Another controversy that has generated much speculation is that of titanium allergy or hypersensitivity. A study by Sicilia et $\mathrm{al}^{5}$ in 2008 considered 1500 dental implant patients, in which an estimated $0.6 \%$ of the subjects were said to be allergic to titanium. The authors concluded that while the prevalence was quite low, a significantly higher risk of positive allergic reaction was found in patients showing postoperative allergy compatible response (ACR), in which cases allergy tests could be recommended. In another study by Muller and Valentine-Thon ${ }^{6}$ in 2006, the authors tested 56 patients who had developed health 
problems, such as muscle and joint pain, chronic fatigue syndrome (CFS), neurological problems, depression, multiple chemical sensitivities (MCS), dermatitis and acnelike facial inflammation after receiving Ti-based dental and/ or endoprosthetic implants. The MELISA ${ }^{\circledR}$ test was performed on all patients before and on 15 patients also after removal of the implants. Of the 56 patients tested in MELISA $^{\circledR}, 21(37.5 \%)$ were positive, $16(28.6 \%)$ ambiguous and $19(33.9 \%)$ negative to titanium. Following removal of the implants, all patients showed remarkable clinical improvement. In the 15 retested patients, this clinical improvement correlated with normalization in MELISA ${ }^{\circledR}$ reactivity. The authors concluded that $\mathrm{Ti}$ can induce clinically-relevant hypersensitivity in certain patients' chronically exposed to it and that Ti should no longer be considered biologically inert.

Even though there are extremely few studies suggesting titanium allergy as a major cause for preloading failure of dental implants, the possibility cannot be discarded. Apart from these, poor compatibility with modern imaging techniques, such as magnetic resonance imaging (MRI) is also an issue.

Typically, metals used for implantation have large elastic modulus (approximately 6-20 times greater than that of the surrounding bone $)^{7-9}$ causing impaired load force transmission at the implant-tissue interface. Consequently, according to Wolff's law this would lead to decreased bone tissue loading that could contribute to stress shielding and peri-implant bone resorption. ${ }^{10,11}$ To overcome these limitations and minimize negative biological reactions, research has focused on designing alternative substitutes to titanium. One of the most promising novel materials is polyetheretherketone (PEEK).

\section{POLYETHERETHERKETONE IN IMPLANTOLOGY}

Following confirmation of its biocompatibility two decades ago, ${ }^{12}$ PEEK was increasingly employed as biomaterial for orthopedic, trauma and spinal implants. PEEK is a relatively new family of high temperature thermoplastic polymers, consisting of an aromatic backbone molecular chain, interconnected by ketone and ether functional groups. The chemical structure of polyaromatic ketones confers stability at high temperatures (exceeding $300^{\circ} \mathrm{C}$ ), resistance to chemical and radiation damage, compatibility with many reinforcing agents (such as glass and carbon fibers) and greater strength (on a per mass basis) than many metals. ${ }^{13}$

Historically, the availability of polyaromatic polymers arrived at a time when there was growing interest in the development of "isoelastic" hip stems and fracture fixation plates with stiffness comparable to bone. ${ }^{14}$ By the late 1990s, PEEK had emerged as the leading high-performance thermoplastic candidate for replacing metal implant components, especially in orthopedics and trauma.
In 1992, PEEK was used for dental applications, first in the form of esthetic abutments and later as implants. Since then many variations in the composition have been carried out to modify and improve upon the working characteristics of the implant.

The implants used in our center had a combination of a phase specific tricalcium-phosphate and titanium dioxide contained within a PEEK matrix. While $80 \%$ of the composite was made of PEEK, $20 \%$ constituted of beta tricalcium phosphate and titanium oxide combined. The $20 \%$ filler content makes the implant isoelastic, i.e. density and elasticity (Young's modulus) identical to bone. Although pure polyaromatic polymers exhibit elastic modulus that varies from 3 to $4 \mathrm{GPa}$, this value can be modified to achieve a modulus close to cortical bone (18 GPa) with the addition of composites. ${ }^{14}$ On the other hand the Young's modulus of titanium and its alloys varies from 110 to $150 \mathrm{GPa} .{ }^{15} \mathrm{It}$ has been proven that a big difference between the elasticity of the implant material and bone leads to greater stress generation due to differential deformation under load. The isoelasticity of PEEK composites ensures that they warp identically to bone and thus produce a more homogenous distribution of stress along the implant bone interface.

The IMI implants (Pornichet, France), which were used in this study were made of the unique $\mathrm{PEEK} / \mathrm{TiO}_{2}$ /beta-TCP combination named BIOPIK ${ }^{\circledR}$. These implants are available in three fundamentally different designs; TAU, THETA and IOTA, for different bone volumes and densities. The 'TAU' is a one piece implant, $4.8 \mathrm{~mm}$ in diameter (10.5, 12 and $15 \mathrm{~mm}$ lengths), designed for insertion and immediate loading in medium to low density bone. 'THETA' has same dimensions as 'TAU' but it is indicated in D1 bone. The 'IOTA' is a $3 \mathrm{~mm}$ diameter implant (lengths 10 and $12 \mathrm{~mm}$ ) that is meant for use in narrow ridges. All these are one piece implants which can be modified intraorally at the time of placement. They have shown good strength, fracture resistance and satisfactory bioinertness. Apart from this, the BIOPIK $^{\circledR}$ material has been shown to promote human osteoblast cell growth and proliferation ${ }^{15}$ making it osteoconductive. However, despite such merits, we felt that the radiographic characteristics of BIOPIK were a cause for concern. Being faintly radiopaque, the implant is not easily visualized on conventional radiographs. While the manufacturers laud this as a significant advantage for someone who might undergo a CT or MRI investigation, its relatively inconspicuous appearance can hinder postoperative radiographic evaluation. Also, the range of diameters and lengths available is rather restricted as compared to most current implant systems.

\section{CASE REPORTS}

\section{Case 1}

A 25-year-old male patient reported to the Department of Oral and Maxillofacial Surgery at Santosh Dental College, 
NCR, Delhi for replacement of missing 11 (Fig. 1). After discussing all options for single tooth replacement, he decided to get implant placement with immediate temporization. Following measurements on an intraoral periapical radiograph (Fig. 2), we planned for a $4.8 \mathrm{~mm}$ (diameter), $12 \mathrm{~mm}$ (length) TAU implant.

The system employs a compact kit with four cylindrical drills, one tapered terminal drill and two knurled knobs used to operate the terminal drill manually (Fig. 3). After establishing the initial depth with two sequential cylindrical drills (Figs 4 and 5), the terminal drill was used manually (Fig. 6). The implant was carried to the osteotomy with a forcep and gently tapped into its final position (Figs 7 to 9). The bone 'shavings' collected in the flutes of the terminal drill were retrieved and placed around the implant collar (Figs 10 and 11). The 'abutment' part of the implant was immediately shaped with a straight surgical handpiece at 45,000 RPM and temporized in the same sitting (Fig. 12). A postoperative IOPA X-ray was taken (Fig. 13). The final prosthesis was delivered at the time of suture removal seven days later (Fig. 14).

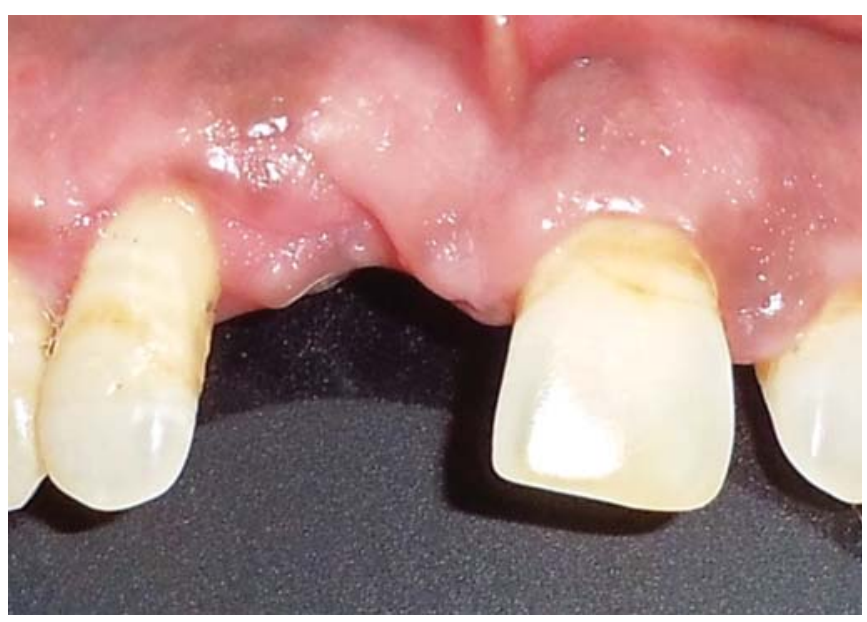

Fig. 1: Intraoral view of missing 11

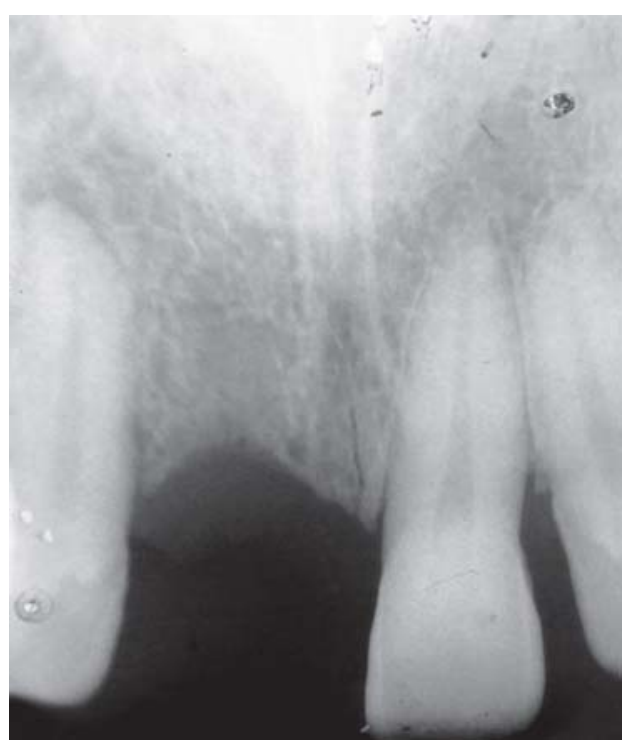

Fig. 2: Preoperative IOPA X-ray

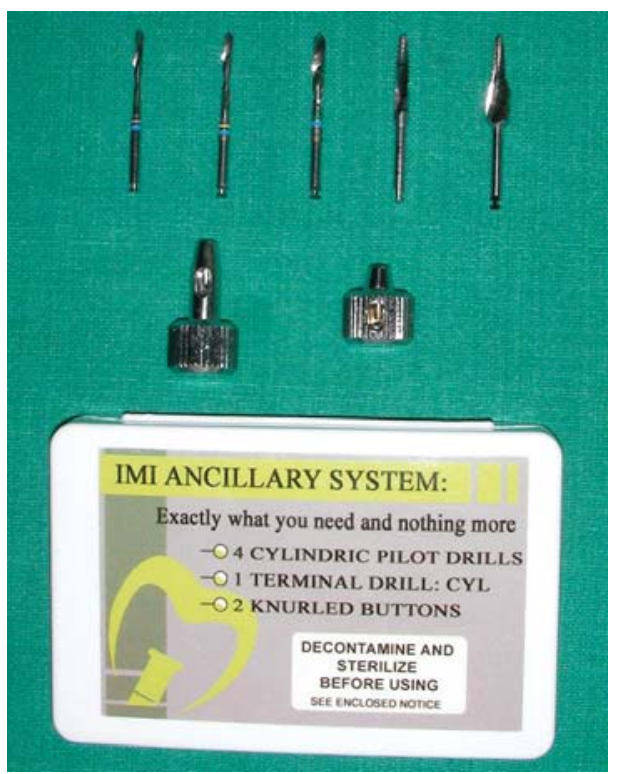

Fig. 3: Kit with drills and knurled knob

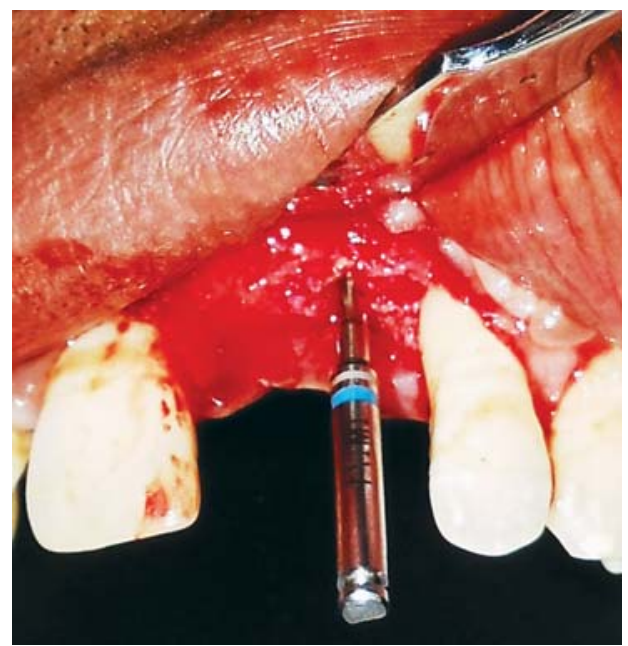

Fig. 4: Establishing initial depth with pilot drill

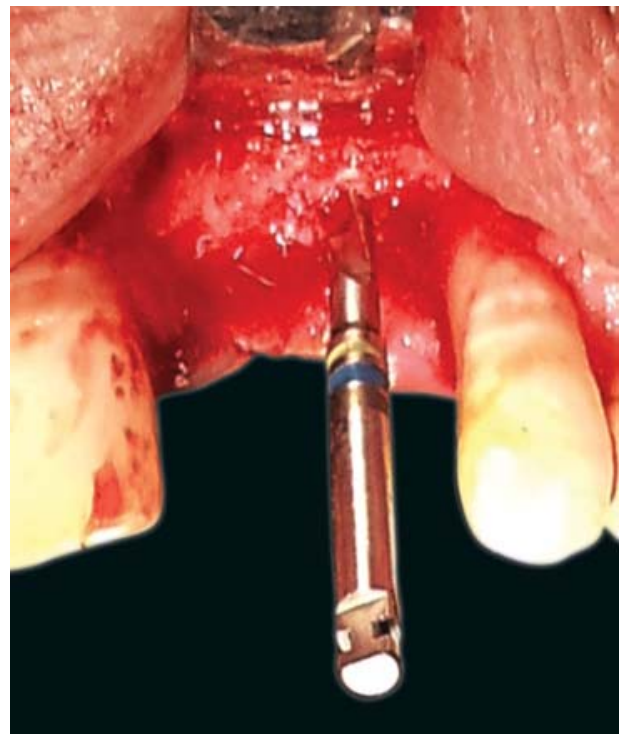

Fig. 5: Second cylindrical drill in place 


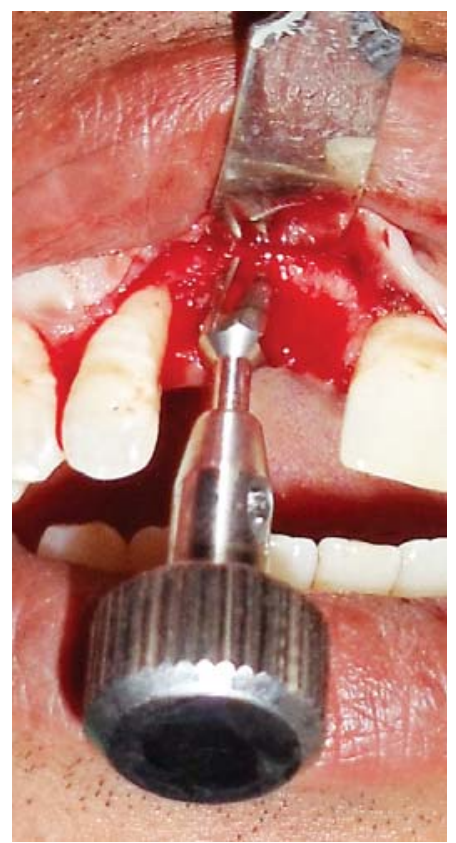

Fig. 6: Terminal drill engaged in the osteotomy with a manual knurled knob

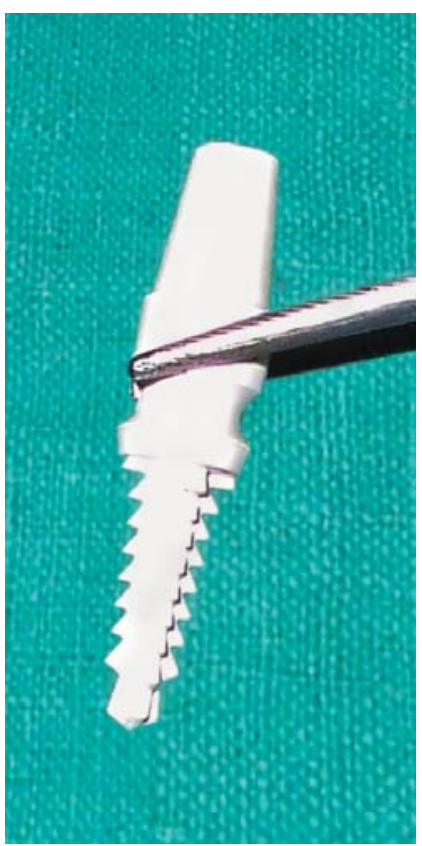

Fig. 7: TAU implant

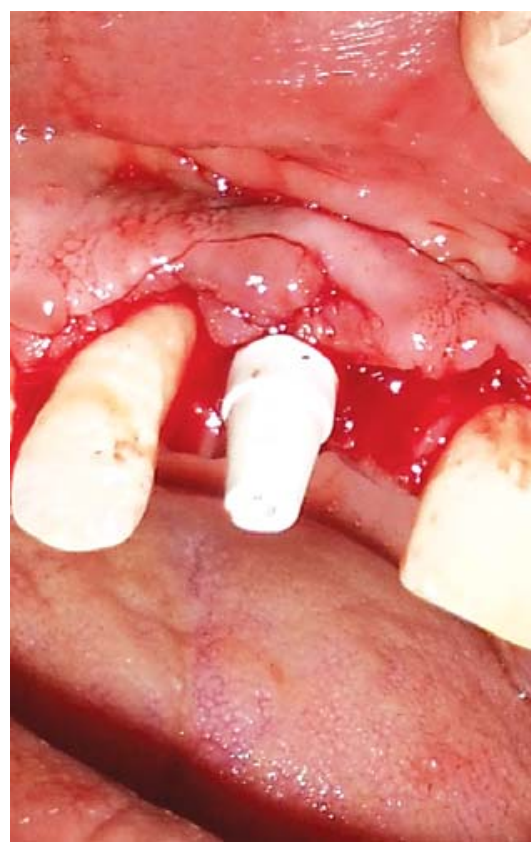

Fig. 8: Implant placed at osteotomysite

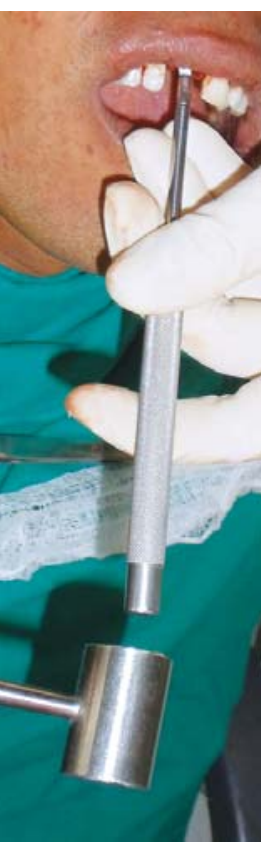

Fig. 9: Soft compac tion of implant into osteotomy

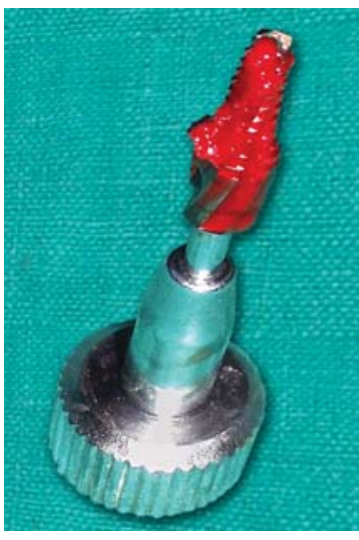

Fig. 10: Bone 'shavings' entrapped in drill flutes

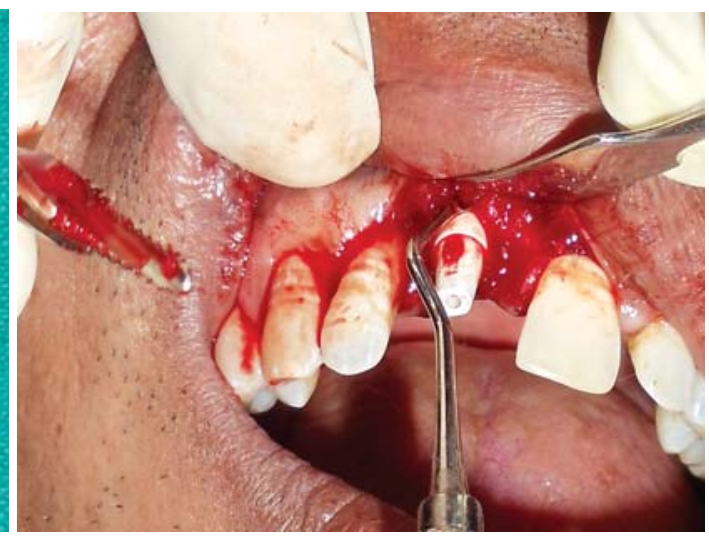

Fig. 11: Placing bone shavings around implant collar

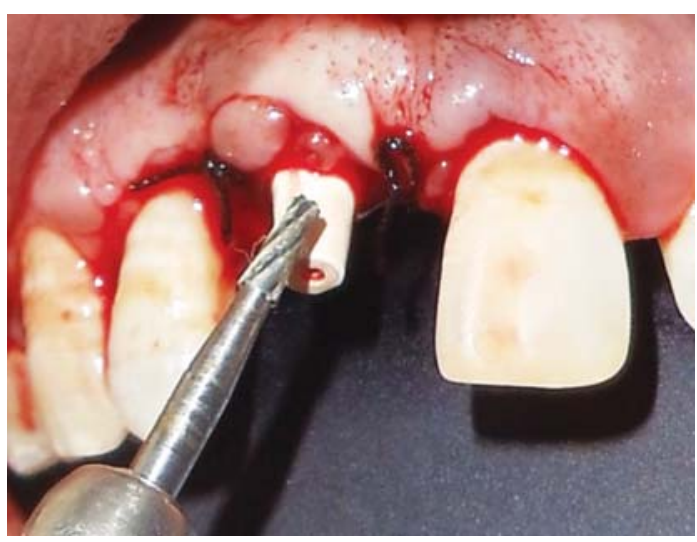

Fig. 12: Preparing the coronal part of the implant

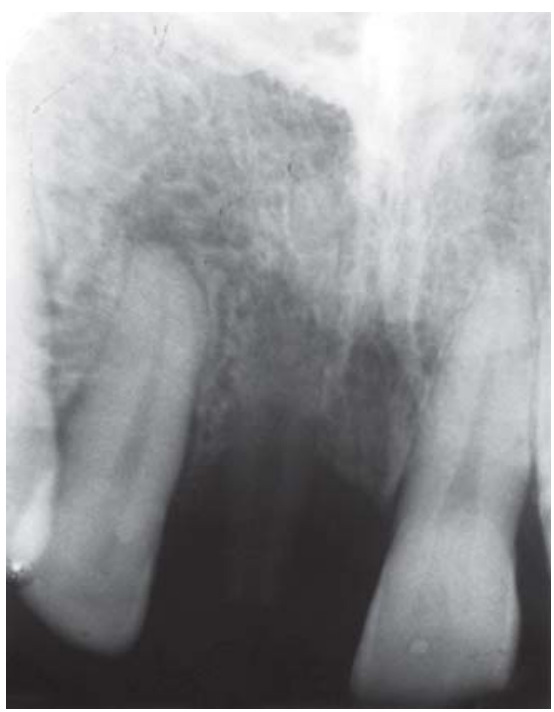

Fig. 13: Postoperative IOPA X-ray showing radiographic character of $P E E K$

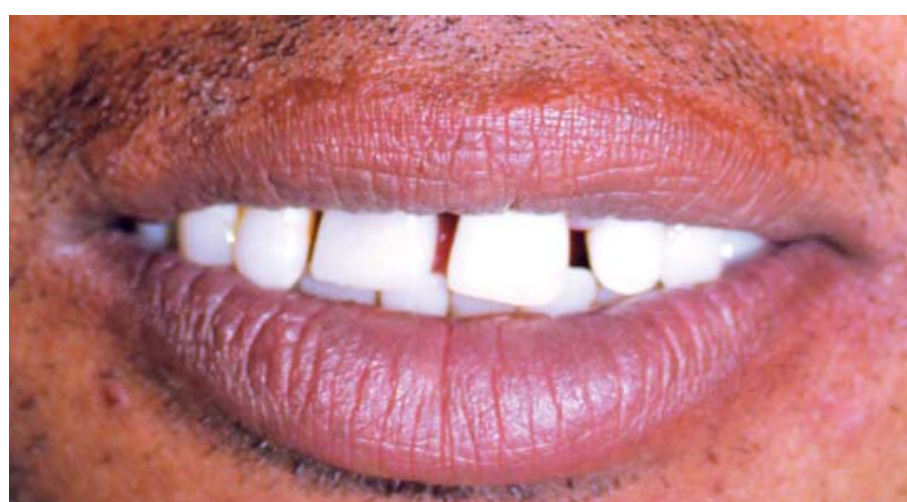

Fig. 14: Extraoral view of final outcome 


\section{Case 2}

A 28-year-old male patient reported to the outpatient department with missing first molar and root stumps of second molar in mandibular right quadrant (Fig. 15). The patient had experience of getting fixed partial denture (porcelain fused to metal) in the mandibular left quadrant and wanted to get implants as he was not satisfied with a long span fixed prosthesis. A $4.2 \mathrm{~mm}$ (d) and $10 \mathrm{~mm}$ (l) titanium implant was placed in the fresh extraction socket of 47 with torque value of $65 \mathrm{Ncm}$ achieved during final tightening and resonance frequency measurement showing ISQ value to be 71 (Osstell ISQ, Integration Diagnostics $\mathrm{AB}$, Sweden).

We placed an IOTA implant $3 \mathrm{~mm}$ (d) and $12 \mathrm{~mm}$ (l) in 46 owing to a narrow ridge (Figs 16 to 18). Both the fixtures were loaded after seven days of initial healing period. At

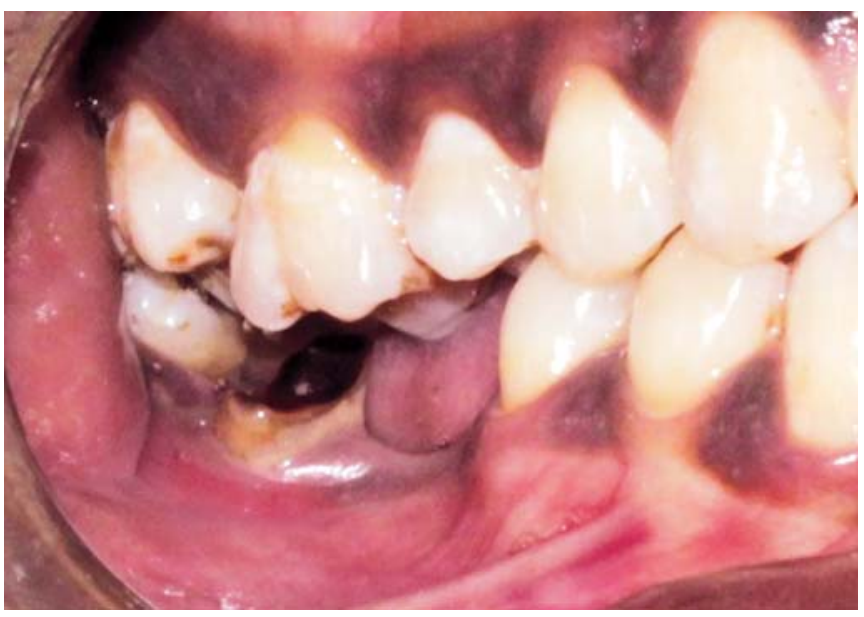

Fig. 15: Intraoral view of root stump in 47 and missing 46

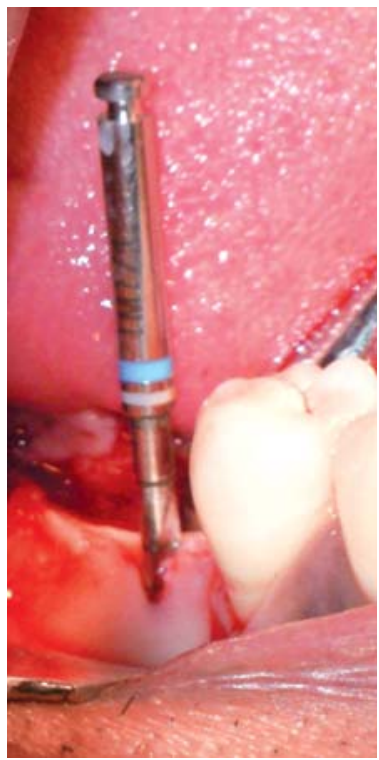

Fig. 16: Cylindrical drill in place

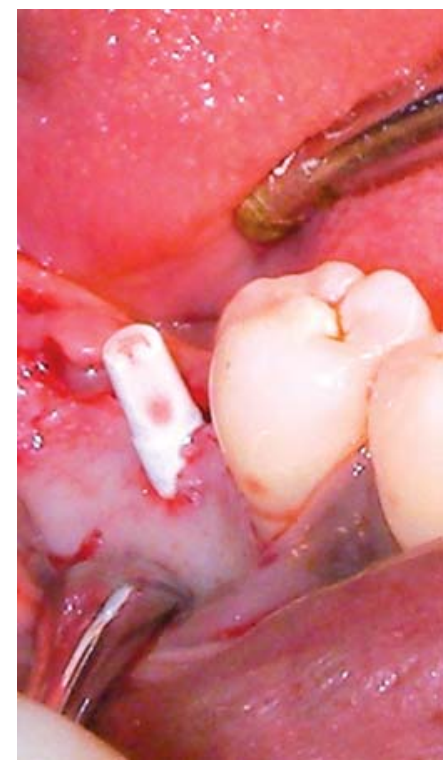

Fig. 17: IOTA implant in place

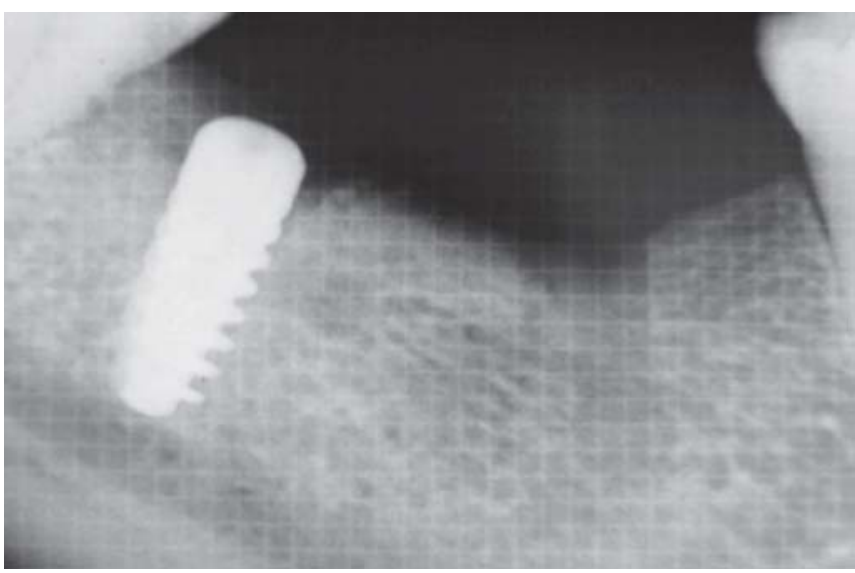

Fig. 18: Postoperative IOPA X-ray showing titanium implant in 47 and PEEK implant in 46

6 months of function, the implant is stable and radiograph shows very little crestal bone loss (Fig. 19).

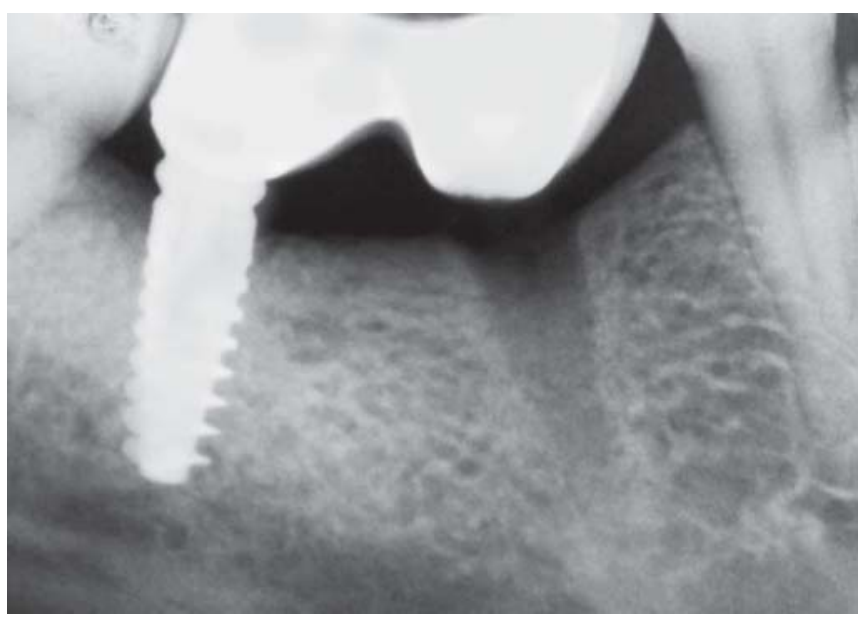

Fig. 19: IOPA X-ray showing 3 months postloading with radiographically apparent increased peri-implant bone density

\section{Case 3}

A 21-year-old female reported to the department of oral and maxillofacial surgery for replacement of missing first molar in mandibular right quadrant. Sufficient width of the alveolar table enabled us to place a $4.8 \mathrm{~mm}$ (d) and 12 mm (l) TAU implant (Figs 20 to 23). In order to make the implant more obviously visible on the radiographs, we inserted a gutta-percha point into a hollow slot that lies in the central axis of the implant. However, we found that this slot does not go all the way upto the apex, and therefore could not function as an indicator to the implant length in the bone. Nevertheless, the gutta-percha point was left in the implant, which was loaded on the 7th postoperative day (Fig. 24).

\section{DISCUSSION}

With the basic structure and function found satisfactory, we believe PEEK implants to have some advantages over 


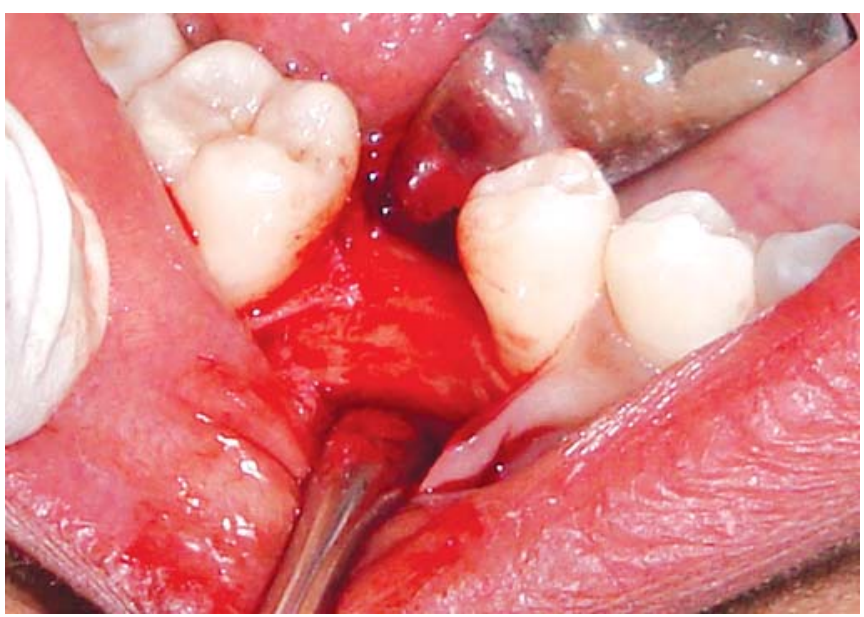

Fig. 20: Intraoperative view of buccolingually adequate alveolar ridge in 46

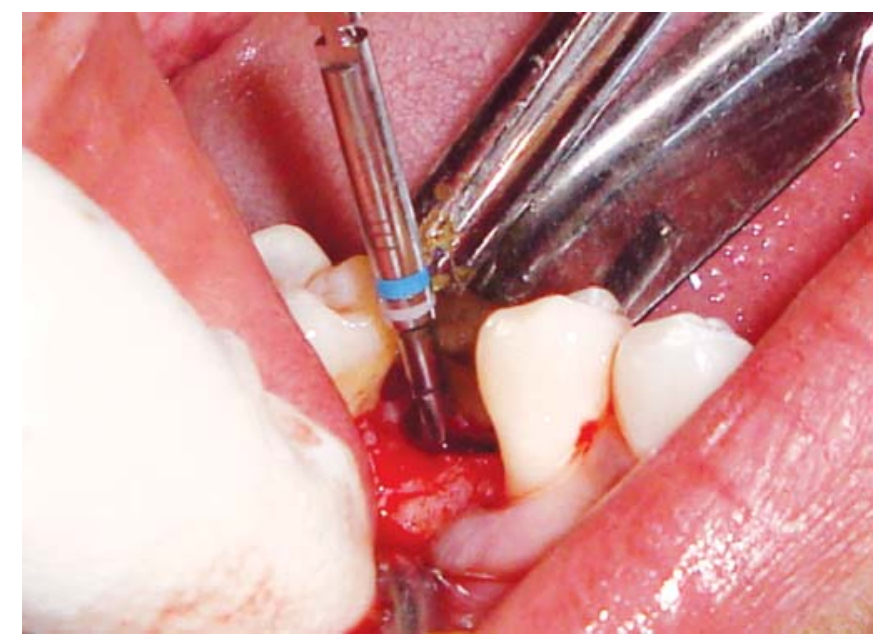

Fig. 21: Cylindrical drill in place

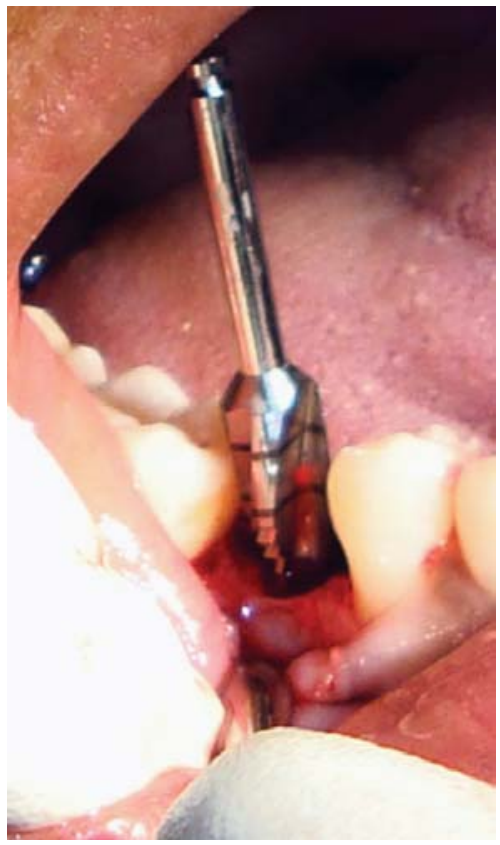

Fig. 22: Terminal drill engaged in the osteotomy

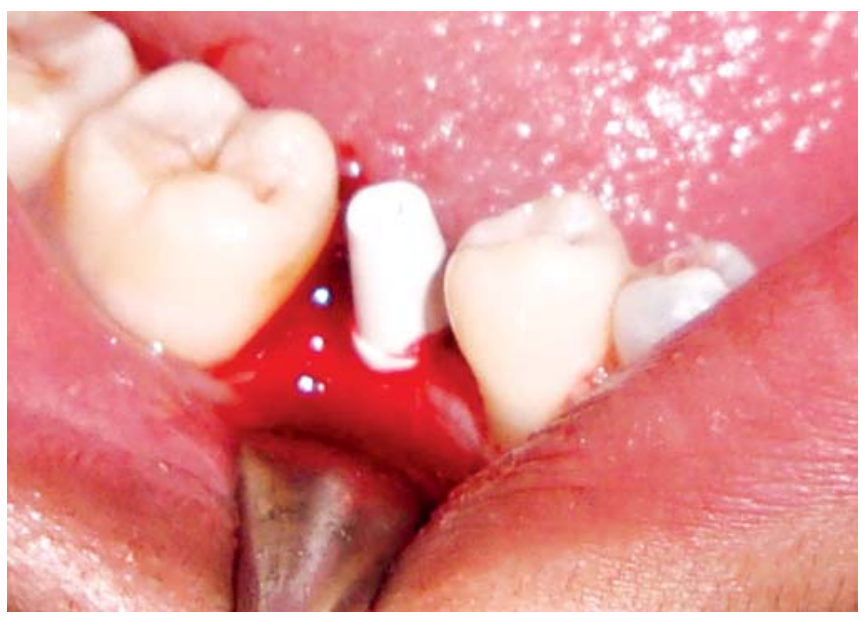

Fig. 23: TAU implant placed at osteotomy site

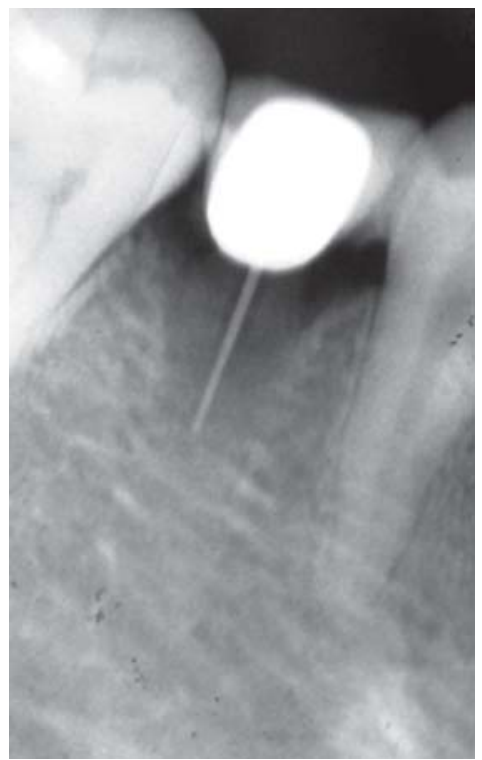

Fig. 24: IOPA X-ray showing functionally loaded PEEK implant with gutta-percha point inserted for localization

titanium. Firstly, their white color makes them ideal for use in the esthetic zone. In our experience, titanium implants impart a grey hue in maxillary anterior region with deficient buccal bone. In some cases with gingival recession, a small part of the implant may become visible, which is highly unacceptable to the patient. Clinicians have gone to great lengths to counter these issues with esthetic zirconia abutments or PEEK abutments later. In the case of PEEK implants, such would not be the case, although a more comprehensive study would be needed to provide definitive data.

Another matter of great convenience was the fact that polymer-composites do not generate heat when they come in contact with a high speed rotary cutting bur. As a result, the coronal portion of the single piece implant could be immediately modified (like crown preparation for FPD) to meet the prosthetic requirement. For example, the height and width of the abutment part of the implant was 
immediately prepared after implantation with a simple straight fissure carbide bur at 40,000 RPM. Even in the anterior esthetic zone, instead of using a two piece titanium implant with angulated abutment we used a single piece PEEK implant and prepared the abutment portion immediately to receive a temporary restoration.

The implant comes with a small simple kit with conventional drills and one hand drill that is used to finalize the osteotomy. This hand drill collects bone shavings that can be collected and used around the implant in immediate extraction cases. The implant is then simply tapped (press fit) into the osteotomy using a mallet.

An important issue that crops up after placing the implant is the fact that it is largely radiolucent, and therefore not clearly visible on a radiograph. There is a slight change in bone density owing to the osteotomy which can be perceived as the space occupied by the implant. However, after 6 weeks in place some amount of radiopaque dense bone can be seen surrounding the implant. This combined with a clear break in the continuity of the bony trabeculae, makes it relatively easy to identify the implant position and signs of crestal bone loss (if any). On the upside, this feature allows us to avoid scatter in further CTs or MRIs, something that has proved to be a great boon for this material in its neurosurgical and orthopedic applications.

As to the isoelasticity of the material giving it any advantage over titanium, there has been one recent study claiming otherwise. ${ }^{17}$ The authors compared the stress distribution of four groups in a Finite Element Model; titanium implant with titanium abutment, titanium implant with PEEK abutment, PEEK implant with titanium abutment and PEEK implant with PEEK abutment. All four were put under identical loads in similar bone. They found that PEEK implants did not present any advantages in relation to the titanium implants regarding stress distribution to the periimplant bone. The study adds that PEEK presents better results than titanium in terms of orthopedic applications as a higher deformation following spine movement increases the stress distribution area and reduces the load concentration. The bonding between implant and bone is rigid in dental implantology, and therefore does not allow for expansion by deformation. Such deformation is inherent in PEEK and presents a different functional behavior that significantly concentrates more stress in the implant neck. It is beyond the scope of this case report to comment on this matter and more research is needed in this direction.

\section{CONCLUSION}

Polyetheretherketone has shown promise in its many forms in medical application. It has osteointegration potential through osteoconduction that has been confirmed by clinical results. PEEK implants were simple to place, functioned well when immediately loaded and possess sufficient merits to warrant further clinical investigation. Their use in the esthetic zone can be of significant advantage to the surgeon as well as patient. However, diminished radiopacity and limitation of available sizes may be causes for concern. Longitudinal studies with large sample sizes and systematic evaluation will provide a more comprehensive view of PEEK dental implants.

\section{REFERENCES}

1. Cortada M, Giner L, Costa S, Gil FJ, Rodriguez D, Planell JA. Galvanic corrosion behavior of titanium implants coupled to dental alloys. J Mater Sci Mater Med 2000;11:287-93.

2. Tschernitscheck H, Borchers L, Geurtsen W. Non-alloyed titanium as a bioinert material: A review. Quintessence Int 2005;36:523-30.

3. Olmedo D, Fernandes MM, Guglidmotti MB, Cabrini RL. Macrophages related to dental implant failure. Implant Dent 2003;12:75-80.

4. Roynesdal AK, Ambjornsen E, Haanaes HR. A comparison of three different endogenous nonsubmerged implants in edentulous mandible: A clinical report. Int J Oral Maxillofac Implants 1999;14:543-48.

5. Sicilia A, Cuesta S, Coma G, Arregui I, Guisasola C, Ruiz E, Maestro A. Titanium allergy in dental implant patients: A clinical study on 1500 consecutive patients. Clin Oral Implants Res Aug 2008;19(8):823-35.

6. Muller KE, Valentine-Thon E. Hypersensitivity to titanium: Clinical and laboratory evidence. Neuro Endocrinol Lett 2006; 27(Suppl 1):31-35.

7. Kitamura E, Stegaroiu R, Nomura S, Mikakawa O. Biomechanical aspects of marginal bone resorption around osseointegrated implants: Considerations based on a threedimensional finite element analysis. Clin Oral Implants Res 2004;15(4):401-12.

8. Rho JY, Ashman RB, Turner CH. Young's modulus of trabecular and cortical bone material: Ultrasonic and microtensile measurements. J Biomech 1993;26(2):111-19.

9. Isidor F. Influence of forces on peri-implant bone. Clin Oral Implants Res 2006;17(Suppl 2):8-18.

10. Steinemann S. Titanium: The material of choice. Periodontology 2000;1998;17:7-21.

11. Huiskes R, Weinans H, van Rietbergen B. The relationship between stress shielding and bone resorption around total hip stems and the effects of flexible materials. Clin Orthop 1992;274:124-34.

12. Williams DF, McNamara A, Turner RM. Potential of polyetheretherketone (PEEK) and carbon-fiber reinforced PEEK in medical applications. J Mat Sci Letters 1987;6:199-90.

13. Kurtz S, Devine J. PEEK biomaterials in trauma, orthopedic, and spinal implants: A review. Biomaterials 2007;28:4845-69.

14. Skinner HB. Composite technology for total hip arthroplasty. Clin Orthop Relat Res Oct 1988(235):224-36.

15. Koca OL, Eskitascioglu G, Usumez A. Three-dimensional finite element analysis of functional stresses in different bone locations produced by implants placed in the maxillary posterior region of the sinus floor. J Prosthet Dent 2005;93:38-44.

16. Harmand MF, Cougoulic JP. A new biocompatible biomaterial: $\mathrm{PEEK} / \beta-\mathrm{TCP} / \mathrm{TiO}_{2}$ composite. Sydney 9WBC Congress 2004.

17. Sarot, Contar, da Cruz, Magini. Evaluation of the stress distribution in CFR-PEEK dental implants by the threedimensional finite element method. J Mater Sci: Mater Med 2010;21:2079-85. 